\title{
A Tactile Sensing Approach in Stroke Rehabilitation
}

\author{
Xinli Du ${ }^{1 *}$, Nikolay Mikov ${ }^{1}$, Amir Mohagheghi ${ }^{1,2}$, Cherry Kilbride $^{1}$, Meriel Norris $^{1}$ and Peter Brett ${ }^{3}$ \\ 1. Brunel University London, U.K. \\ 2. University of Social Welfare and Rehabilitation Sciences, Tehran, Iran \\ 3. University of Southern Queensland, Toowoomba, Australia
}

Corresponding to Xinli Du, xinli.du@brunel.ac.uk, Brunel University London, U.K. UB8 3PH

\begin{abstract}
The paper describes an experimental, mechanically simple, tactile sensing solution in the form of a sensing chair for discriminating human motion in a reaching task. This costefficient technical approach was employed for the assessment of selective arm movements in stroke survivors. The sensing system classifies trunk motion in a seated stroke survivor during a goal-directed task where there is direct correlation with the level of severity of arm movement. The system interprets motion mechanically from coupled sensory data transients using artificial neural networks and shows tolerance to patients' sitting posture and performance variability. The accuracy of classification was typically greater than $94 \%$ across three categories when applied to a group of stroke survivors of wide-ranging motor abilities. The mechanical simplicity, versatility of approach for use in other classes of movement, and potential low cost of manufacturing provides opportunity to employ the system at clinics and homes for assessment and training.
\end{abstract}

Key words: Distributive Tactile Sensing, Stroke Rehabilitation, Upper Limb Kinematics

\section{Introduction}

Stroke and its associated motor impairments are important health problems. Within the UK, 152,000 new cases are reported annually, and the number of stroke survivors is currently at 1.1 million [1]. Movements of both upper and lower extremities can be impaired by various degrees of severity after stroke. This can negatively affect survivors' independence and quality of life and finding new therapeutic interventions and assessment techniques remain the foci of research investigations in this population.

Functional movements are encouraged during rehabilitation, and following principles of motor learning, movement repetition, and accurate and timely feedback remain the cornerstone of every training session to improve function. To restore functionality, successful movement must be reinforced regularly at both clinic and home. The enabling solution should therefore be cost efficient, easy to set up, and provide feedback that can be readily understood and used by the user. In contrast with other research assisting repetitive motion therapy [2 4], the present study provides a solution to these problems by providing a means to discriminate motion of the trunk, which is correlated to the severity of arm movement, during a reaching task, and is devised in the form of a seat using tactile information to discriminate motion.

In biomechanics and motor control research, a common method of motion detection is via vision motion analysis systems [5,6] and examination of the forces applied to a transducer during movement $[7,8]$. For example, a force plate, which is frequently used in gait analysis, determines components of the load vector applied to the plate during walking/running, and position of application of the ground reaction force in the form of centre of pressure [10,11]. In other applications, force and pressure are used in the precise control of machines [10], toys and consumer devices, and bioengineered systems such as prosthetics [11], exoskeleton devices [11] and surgical tools $[12,13]$.

The sensing method explored in this study was in the form of a mechanically simple instrumented surface which replaced seat of an office chair and used to retrieve motion information in real-time from a sitting participant performing a reaching task. Simultaneous 
time series data was retrieved from a few sensing positions that was mechanically coupled through the deformation of the surface of contact with the person. Features automatically identified from the coupled data transients are used to discriminate the nature or amplitude of motion in the task performed. The approach is often termed the 'Distributive approach to tactile sensing'.

Generally, tactile sensing is often applied in point load measurement [9-11], whereas in practice there is much more information to be retrieved if spatial distribution and transients of forces are taken into consideration. The distributive approach applied to tactile sensing has been explored extensively by the research team. It assumes that, for a specific task, loading occurs somewhere in the spatial range of a deforming structure and that events are recognized through the expression of a coupled set of sensing elements rather than specific values. The coupling of sensory transients through the deforming structure enables recognition of changing loading with sensitivity and without a specified location. The approach enables a mechanically straightforward setup to find the point of application, the nature or distribution of the load and loading transient. This is correlated with the event of interest. In contrast to sensor arrays [14] which utilise a high number of sensing elements to achieve high spatial resolution, the distributive approach would require only few sensing elements ( 3 or 4 ), with resolution of the system principally attributed to digital conversion of sensory outputs rather than physical separation of elements. It is applied readily where the goal is to identify or contrast as opposed to measure. Examples are surgical robotic tools, where tactile sensing is used to recognize invisible tissues and tissue structures ahead on the tool path by using the tissue as the coupling medium [15], endoscopes [16], catheters, earlier investigations on controlling robotic grippers for handling deforming mediums such as food and dough [17], discriminating cells [18] and in the discrimination of types of gait mentioned earlier [19]. In the latter three applications, the sensing medium has been an artefact, and often a flat plate to which low number of sensing elements have been deployed to enable robust sensing over the full extent of the deforming medium. For applications in discriminating human motion, the approach is tolerant to the build of the person and position of the person on the sensing medium [19 - 21]. Distributed Tactile Sensing (DTS) can therefore be ideally applied to the imprecise expression of multi-dimensional, variable human movement in a specified task. Moreover, information derived from the sensory input can be used to feedback on movement in real-time.

Application of DTS for the assessment of movement in a reaching task in stroke survivors was the focus of the present study. As stated before, in stroke survivors, magnitude of trunk motion is correlated to the severity of arm movement during the reach, and therefore, the aim of this study was to automatically grade the impairment of the selective arm moments in stroke survivor based on the associated trunk motion. The approach was tolerant to the variable seated position, and it was further found that the relevant measurands could be determined using a simplified version of the method that averages sensory outputs. The pragmatic version presented here contributes to the setup of a cost-effective configuration. Anticipated, coupled features in the data were recognized using an algorithm to discriminate the type, extent and changes in movement. Using this information, a near real-time display can be provided for the user and therapist.

\section{Material and methods}

\subsection{Sensing system and the reaching task}

For the reaching task, the sensing medium used is the seat of the office chair shown in Figure 1 , consisting a rigid steal frame with cushioned bottom seat and back support. Proximity sensors were used to determine the deflection of the supporting surface simultaneously. Sixteen sensors were placed across the seat in the experimental construction shown in Figure 2. This distribution enabled the sensitivity of sensor placement on the seat to the performance achieved. Sensing elements were placed left-right symmetrically. In practice only 4 elements 
were used together in the sensing approach used. These are sensors 2, 5, 10, and 13, shown in Figure 2. It was found that a higher density of positions at the rear of the seat enabled exploration of higher sensitivity to discriminate the measurands from variations in expression of the motion in stroke recovery. Vishay-CNY70 IR displacement transducers were used in the chair. The range of the sensors used is limited to displacements of between $3 \mathrm{~mm}$ to $16 \mathrm{~mm}$ (Figure 3).
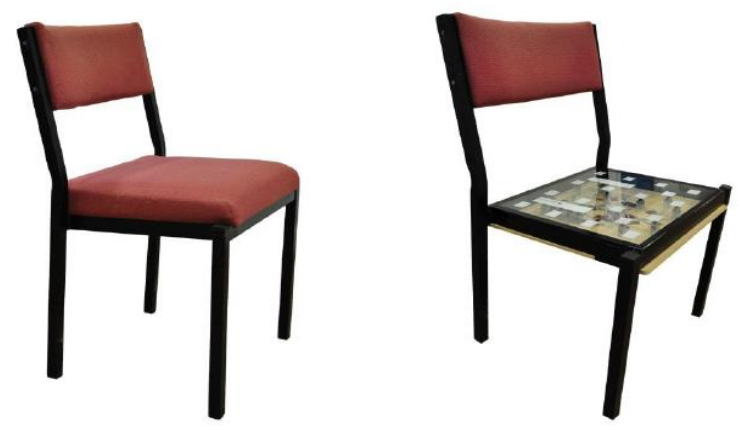

Figure 1 Converted sensing chair

Back of chair seat

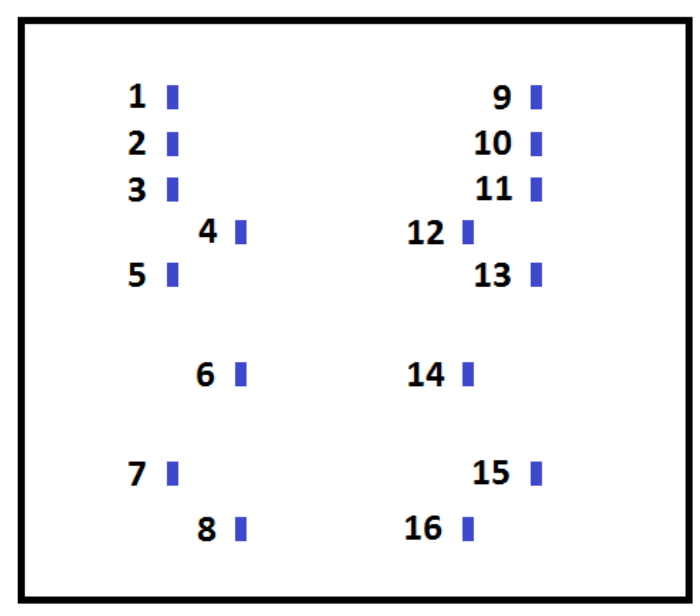

Front of chair seat

Figure 2 Position of sensing elements in the experimental sensing chair

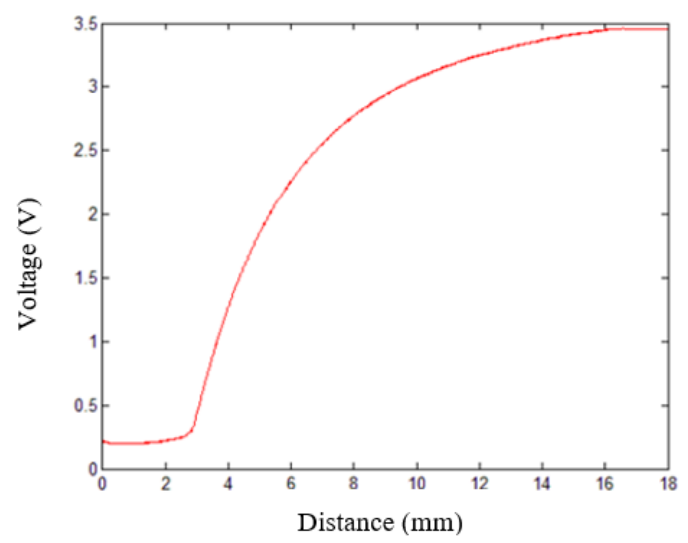

Figure 3 Voltage-Displacement characteristics of IR displacement sensor.

The reaching task (reaching to a target while sitting) is used in therapy to test the fundamental ability of the central nervous system to integrate multiple degrees of freedom (joints, body segments) in motion. In healthy individuals, arm and trunk movements are coordinated when 
reaching to a target placed within $90 \%-100 \%$ of maximum arm reach. The trunk does not contribute to the movement. In reaching, the contribution of the trunk to arm movement is necessary beyond the singularity of maximum arm reach. The movement of the trunk starts before, or simultaneously, with the reaching motion, and finishes after the desired reach is achieved. This pattern of coordination between the arm and trunk is disrupted after stroke leading to trunk recruitment in executing the reach to a target placed within the reach of the arm. Muscle weakness, spasticity, and abnormalities in the regulation of spinal reflexes are amongst suggested mechanisms that likely contribute to the impaired control of movement after stroke.

To discriminate trunk angles and the contribution to the reaching task, healthy subjects were tasked with reaching a target placed at three distinct locations beyond the arm length. Motion was performed using maximum contribution from the arm and different contributions from the trunk (minor, moderate and maximum). In order to unify the performance within the reaching task of these healthy volunteers, targets were placed at distinct locations defined based on their individual maximum arm length. This length was measured from the acromion to third metatarsal anatomical landmarks. Using this metric, the full length of the arm corresponds to $120 \%$ of this distance. Figure 4 illustrates the three discriminative conditions corresponding to reaching to a target placed at distances equal to: $120 \%$ of the arm length involving no or minimal trunk contribution (Figure 4A); $150 \%$ of the arm length involving moderate trunk contribution (Figure 4B); the maximum reach of the participant involving maximum contribution from the trunk (Figure 4C).

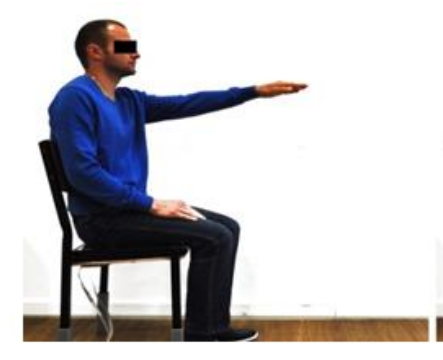

A

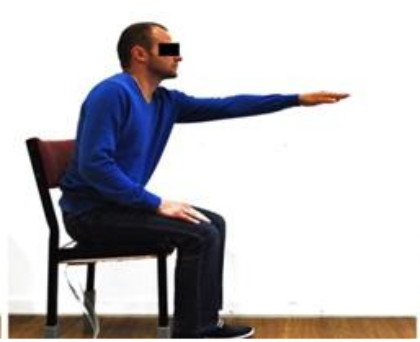

B

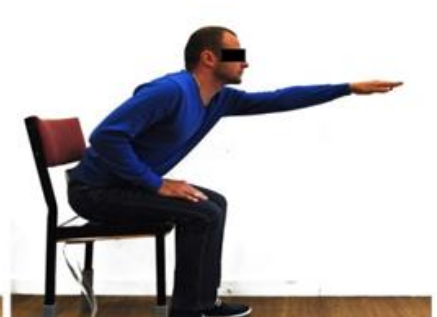

C

Figure 4 Reaching task conditions involving arm contribution and different target location

Using the sensing approach, it is possible to interrogate a variety of asymmetric behavior, however studies showed that for this application by simply averaging the four coupled sensory outputs that the final output was particularly sensitive. It is also helpful to make the algorithm robust to the position of the sitting. Typical sensing transients of the three conditions from one healthy volunteer are shown in Figure 5 respectively. The transients represent the deflection of the surface. For condition A (120\% arm length), it can be observed that the sensing transient has low amplitude and, as would be expected, there is increased amplitude with increased reach, and consequently greater trunk contribution to the motion. In all three conditions, sensing transients show a down-drift characteristic (indicated in Figure $5 \mathrm{~B}$ ) that is associated with initiation of trunk bending. In condition $\mathrm{B}$ and $\mathrm{C}$, the peak amplitude of the deflection corresponds to the relaxation by the participant having reached the target and there is acceleration back to the initial position. In all three transients, it is also observed that there is an up-drift stage (indicated in Figure 5B) when the trunk is accelerating back to the initial position. 

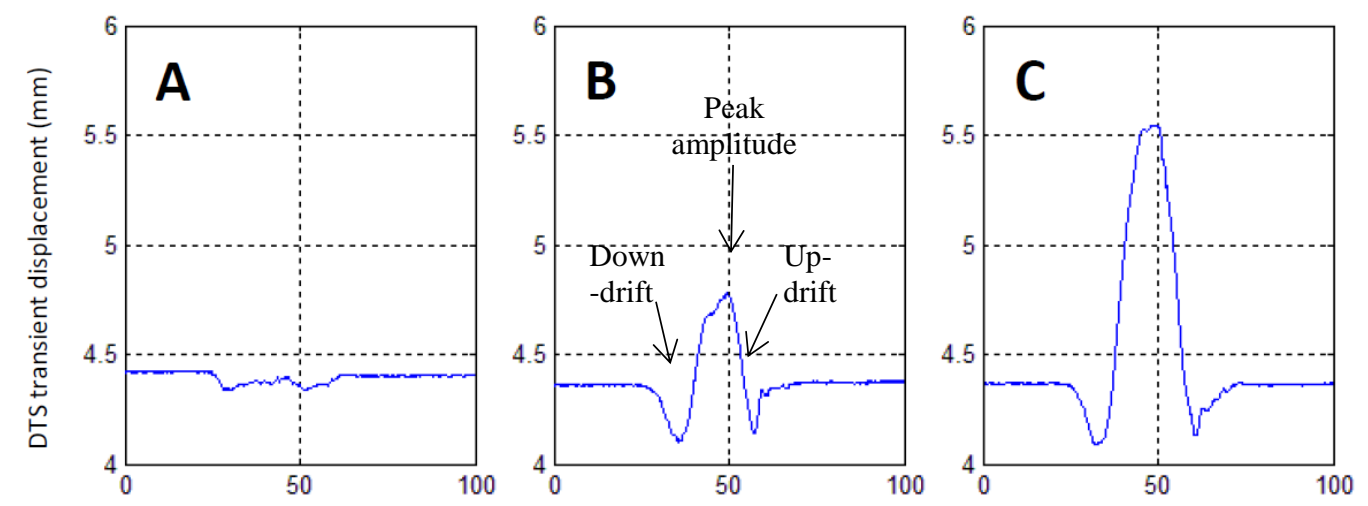

Normalised in percentage time $(0-100)$

Figure 5 Sensing transients during the reaching tasks under condition A: 120\% of the arm length, B: $150 \%$ of the arm length, and $\mathrm{C}$ : the maximum reach

\subsection{Training of the artificial neural network}

A simulation model is introduced to generate data for an Artificial Neural Network (ANN) training to discriminate different reaching conditions. Such a model would provide a more comprehensive understanding of the behaviour of the DTS system. Furthermore, it provides an opportunity for developing more advanced neural networks that could be applied to a wider range of individuals with different anthropometric characteristics that could affect behaviour of DTS transients. Already, it is not unusual to train an ANN with a dataset which is artificially enlarged using label-preserving transformations, or training models solely on synthetic data, such as the ground-breaking work on text recognition in the wild [22-24]. The advantage of using the training model can help to improve the robustness of the dataset. This has been demonstrated [25, 26]. In this work, a 2-linked dynamic body model (based on Lagrange principle) consisting of a trunk and an arm segment was developed. The initial positioning of the model linked segments was approximated according to a normal sitting posture with a narrow trunk and straight arm pointing to the relevant knee as shown in Figure 6. Motion of the arm segment (link2) for the three reaching conditions was defined by rotation of that segment around joint 2 from its initial position to its forward horizontal positioning. Motion of the trunk segment (link1) was defined according to the reaching distances based on the participant's arm length. Therefore the simulated conditions corresponded to reaches involving maximum arm contribution and different levels of contribution from the trunk relevant to the ones performed in the real situation.

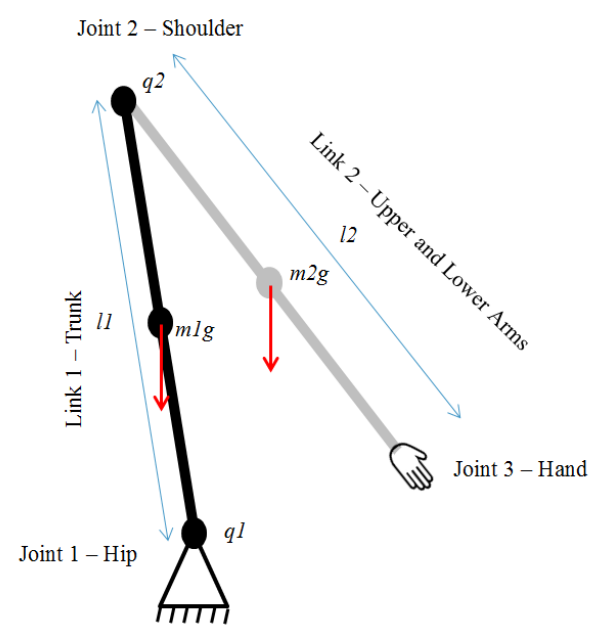

Figure 6 The 2-linked segment model consisting of a trunk segment (link 1); an arm segment (link 2); hip joint (joint $1-\mathrm{q} 1$ ); shoulder joint (joint $2-\mathrm{q} 2$ ); and hand joint (joint 3 ). 
Masses and lengths of the simulated body segments were scaled according to [27]. Inverse dynamics was used to calculate simulated 2-dimensional vertical forces applied to the seat during the reach and associated simulated DTS transients. The dynamic motion simulated by the system was 1.5 seconds long and included the return phase that brought the system back to its initial position (i.e. $0.75 \mathrm{~s}$ for each of the forward and backward phases). For simulating DTS device transients, a FDM (Finite Difference Method) plate model was developed. Input forces to the FDM plate used in the simulations were defined as sum of two components: 1 a static force of $480 \mathrm{~N}$ magnitude (corresponding to approximately $70 \%$ of the mass of a $70 \mathrm{~kg}$ person applied to the seat of the chair while sitting); 2 - a vertical dynamic force generated by the 2-linked body model and discretised with a sampling rate of $50 \mathrm{~Hz}$. The sequence of the discretised dynamic force that was applied to the simulated DTS model was subjected to the static uniformly distributed input force above in order to simulate the behaviour of the system in a quasi-static condition. The deformation characteristics of the simulated DTS device were approximated with that of the real DTS device by choosing a Young's modulus of $1 \mathrm{GPa}$, which was approximated from the range of acrylic materials [28]. The comparison between the collected data and the simulated results are shown in Figure 7.
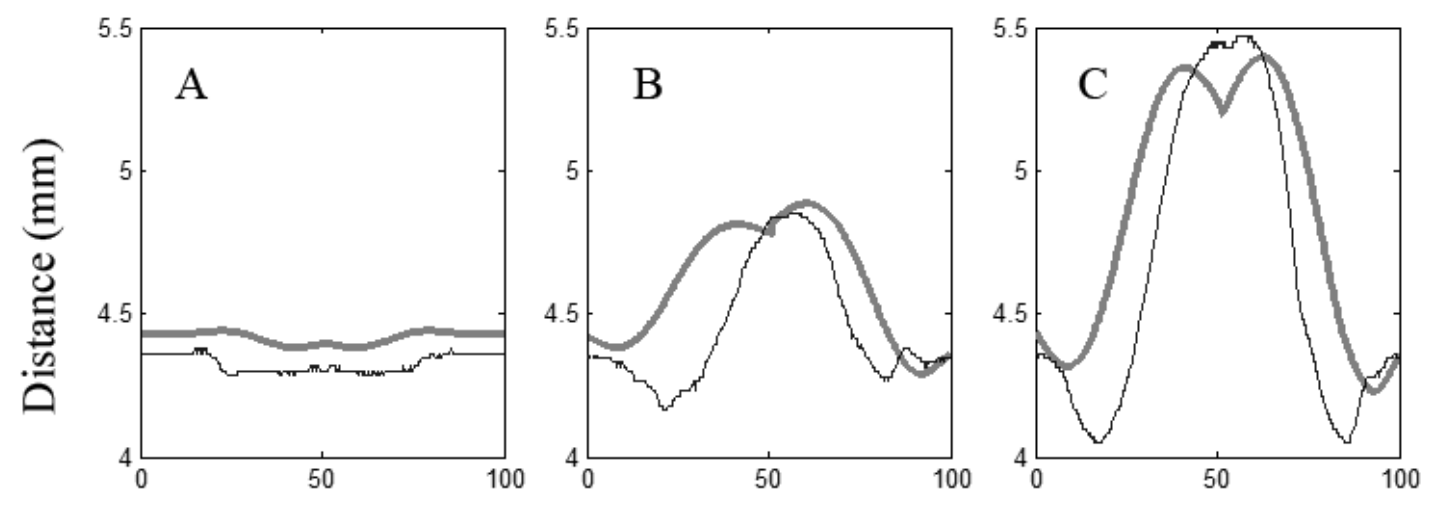

Normalised Time (\%)

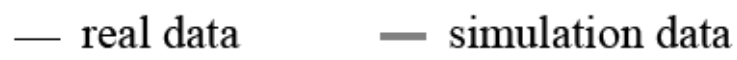

Figure 7 Comparison between DTS transients of Volunteer A obtained based on real (thin) and simulated (thick) data: A - 120\% arm length; B - 150\% arm length; C - Maximum reach

As indicated previously, simulated conditions corresponded to reaches involving maximum arm contribution and different levels of contribution from the trunk relevant to the ones performed in the real situation (Figure 4). Shapes and amplitudes of the simulated transients (thick lines) were similar to real ones (thin lines). The minimum and maximum amplitudes for both simulated and real transients are summarized in the table below. The error was calculated less than $4 \%$ as shown in Table 1.

\begin{tabular}{|c|c|c|c|}
\hline & Condition A & Condition B & Condition C \\
\hline Min amplitude (real) & 4.27 & 4.17 & 4.06 \\
\hline Min amplitude (simulation) & 4.39 & 4.28 & 4.22 \\
\hline Difference & $2.7 \%$ & $2.6 \%$ & $3.8 \%$ \\
\hline Max amplitude (real) & 4.35 & 4.83 & 5.5 \\
\hline Max amplitude (simulation) & 4.44 & 4.89 & 5.39 \\
\hline Difference & $2 \%$ & $1.2 \%$ & $2 \%$ \\
\hline
\end{tabular}

Table 1 The comparison between real and simulation results 
This indicated that the generated dynamic model could provide appropriate representation of contact forces produced by the moving body in performing reaching tasks. Simulation transients of the $150 \%$ and "maximum reach" conditions, showed a decrease of the transient at the final stage of forward body movement (i.e. at $50 \%$ of the normalised time), and an increase of the transient amplitude at the initial stage of return to the initial position. The two stages were associated with the deceleration and acceleration of the trunk at the middle stage of the reaching task when the trunk stopped to move (at normalised time of 50\%) and started to move backwards, respectively. These features were less noticeable in the transients obtained from real data due to the natural way of performing the reaching task in which the relevant deceleration and acceleration were less pronounced. Similarly, the downward drift associated with the reach initiation stage was noticeably less pronounced in the simulated transients compared with the real ones. For real data this was more noticeable due to the use of chair back support on which the trunk was resting prior to executing the task. Perhaps, when the execution of the task began, the trunk exerted vertical forces affecting the deformations of the distributive surface.

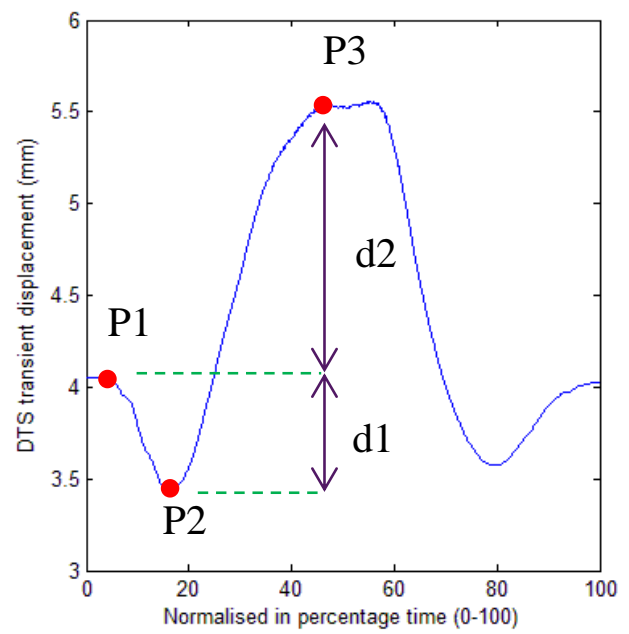

Figure 8 The extracted points and parameters for ANN training

It is observed that the peak to peak amplitude correlates with the amplitude of trunk bending in the task. Using this feature to describe trunk motion in reaching, five parameters; the absolute amplitudes P1, P2, P3, and the differentials d1 = P1(y) - P2(y), d2 = P3(y) - P1(y), shown in Figure 8 are used as the input patent to ANN. MATLAB Neural Network Toolbox was used for developing and training the ANN, having two-layer feed-forward architecture with sigmoid hidden and output neurons. The performance of ANN neural network was tested with 8 neurons on the hidden layer, using scaled conjugate gradient back-propagation learning algorithm.

\section{Calculation}

A clinical study was formed to verify the performance of the sensing chair when subjected to greater variation in presentation of the motion of the trunk and arm from the norm. A motion capture system (Motion Analysis, USA) was used to record the motion of participants.

Markers were placed at the neck, shoulders, elbow, wrist, knuckle, and hips, illustrated in Figure 9. The data retrieved is presented as bending angles for the trunk contrasted with the tactile sensing system predictions from the seat of the chair. 


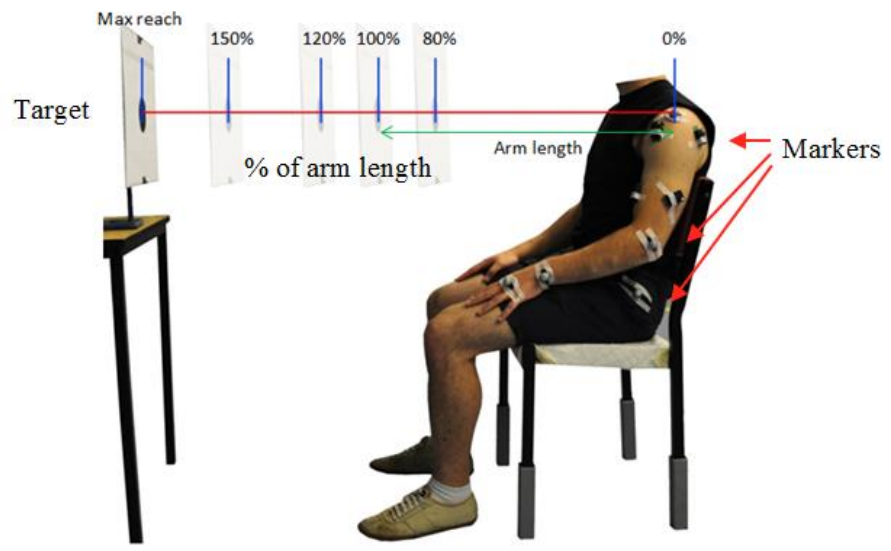

Figure 9 Setup for the pilot clinical study

A further ten healthy individuals and five chronic stroke survivors were included in this study. The physical relevant statistics of the volunteer participants in this study are presented in Table 2. The participants cover both genders and a wide range of body build in terms of weight (48kg to $85 \mathrm{~kg}$ ) and height $(155 \mathrm{~cm}$ to $183 \mathrm{~cm})$. For the healthy individuals, the target is located at the same position as introduced in Figure 4. The identical ANN introduced in previous section was tested for classification of the reaching. The stroke survivor participants are at differing levels of impairment for the motion of the upper limb based on Fugl-Meyer (FM) upper extremity test score (summarized in Table 2). The target to be reached was placed at different locations according the maximum reach of each participant respectively. For the stroke participants who can reach beyond their arm length (S2-S5), the target was placed at a critical boundary which is equal to their arm lengths. For the participant who could not reach an arm length (S1), the target was placed at the maximum reach position, measured as $63 \%$ of arm length. The contribution of trunk movement is identified using the same ANN introduced in section 3 .

\begin{tabular}{|c|c|c|c|c|c|c|c|c|}
\hline $\begin{array}{l}\text { Volunteer } \\
H-\text { Healthy } \\
S-\text { Stroke }\end{array}$ & Gender & Age & $\begin{array}{l}\text { Weight } \\
(\mathbf{k g})\end{array}$ & $\begin{array}{l}\text { Height } \\
\mathbf{( c m})\end{array}$ & $\begin{array}{l}\text { Side } \\
\text { tested }\end{array}$ & $\begin{array}{l}\text { Arm } \\
\text { length } \\
(\mathbf{c m})\end{array}$ & $\begin{array}{l}\text { Max. } \\
\text { reach } \\
(\mathbf{c m})\end{array}$ & $\begin{array}{l}\text { Fugl- } \\
\text { Meyer } \\
\text { score }\end{array}$ \\
\hline H1 & Male & 28 & 85 & 183 & Right & 71 & 132 & N/A \\
\hline H2 & Male & 27 & 84 & 170 & Right & 67 & 115 & N/A \\
\hline H3 & Female & 25 & 48 & 160 & Right & 59 & 111 & N/A \\
\hline H4 & Female & 26 & 67 & 165 & Right & 65.5 & 121 & N/A \\
\hline H5 & Male & 26 & 66 & 170 & Left & 63.5 & 124 & N/A \\
\hline H6 & Male & 78 & 72 & 176 & Right & 68.5 & 118 & N/A \\
\hline H7 & Female & 53 & 63 & 164 & Right & 70 & 122 & N/A \\
\hline H8 & Male & 57 & 78 & 187 & Right & 74 & 133 & N/A \\
\hline H9 & Male & 51 & 83 & 178 & Right & 67 & 125 & N/A \\
\hline H10 & Male & 39 & 82 & 180 & Right & 71 & 129 & N/A \\
\hline S1 & Female & 52 & 50 & 160 & Right & 68 & 43 & 20/60 \\
\hline S2 & Male & 28 & 80 & 178 & Left & 71 & 127 & $58 / 60$ \\
\hline S3 & Female & 56 & 54 & 168 & Left & 65 & 120 & $59 / 60$ \\
\hline S4 & Female & 48 & 57 & 155 & Left & 62 & 106 & $59 / 60$ \\
\hline S5 & Male & 49 & 83 & 175 & Right & 69 & 98 & $38 / 60$ \\
\hline
\end{tabular}

Table 2 Physical records of the healthy and stroke volunteers 


\section{Results}

One result of a 'Max reach' by a healthy participant derived both from the tactile sensory transients and the body bending angles are contrasted in Figure 10. The result confirmed that the tactile sensory transient is sensitive to trunk bending motion in executing the reaching task (as indicated as start and end of trunk bending). It is also shown that both shoulder and elbow angles share similar pattern with the trunk bending angle transient, although the motion of these joints is completed ahead of trunk bending.

One result from a stroke participant (S1) corresponding to a 'max reach' is shown in Figure 11. Both the seat sensing transient and body motion data were presented. It can be found that the seat sensing transient is synchronous to trunk bending as indicated at the start and end of trunk bending. Contrasting with the result from healthy participants, the data of shoulder and elbow angles illustrate the impaired arm movement associated with a hemiplegic pattern of the stroke participant. The DTS sensing transient is fundamentally same as the transient of healthy participant, as explained in section 4.

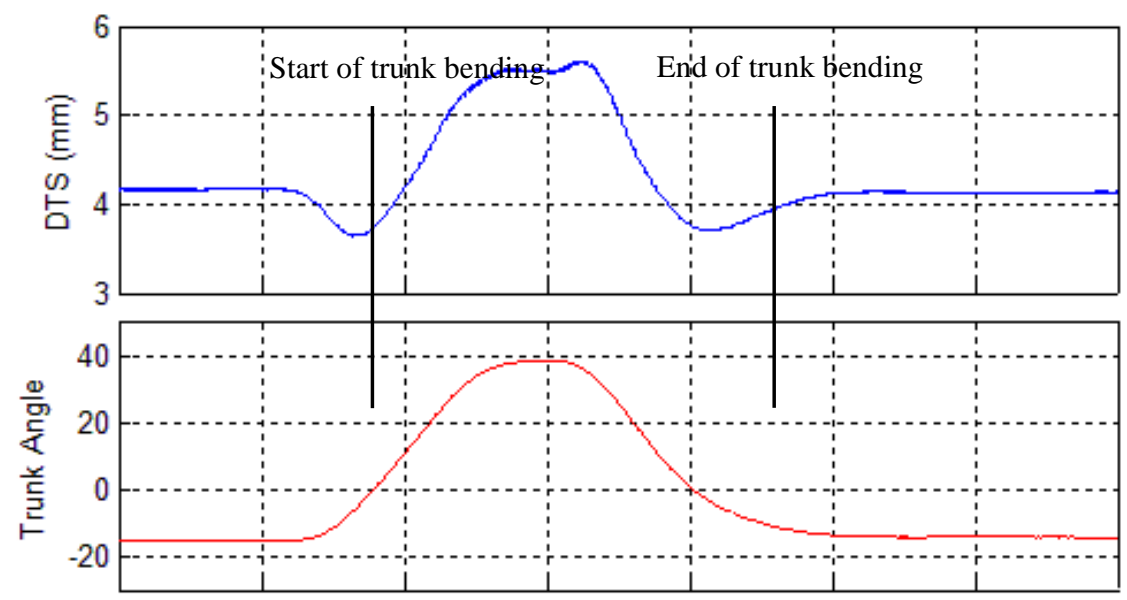

Figure 10 Time normalised DTS transient, motion captured body angles of one healthy participant corresponding to a 'max reach' in the reaching task

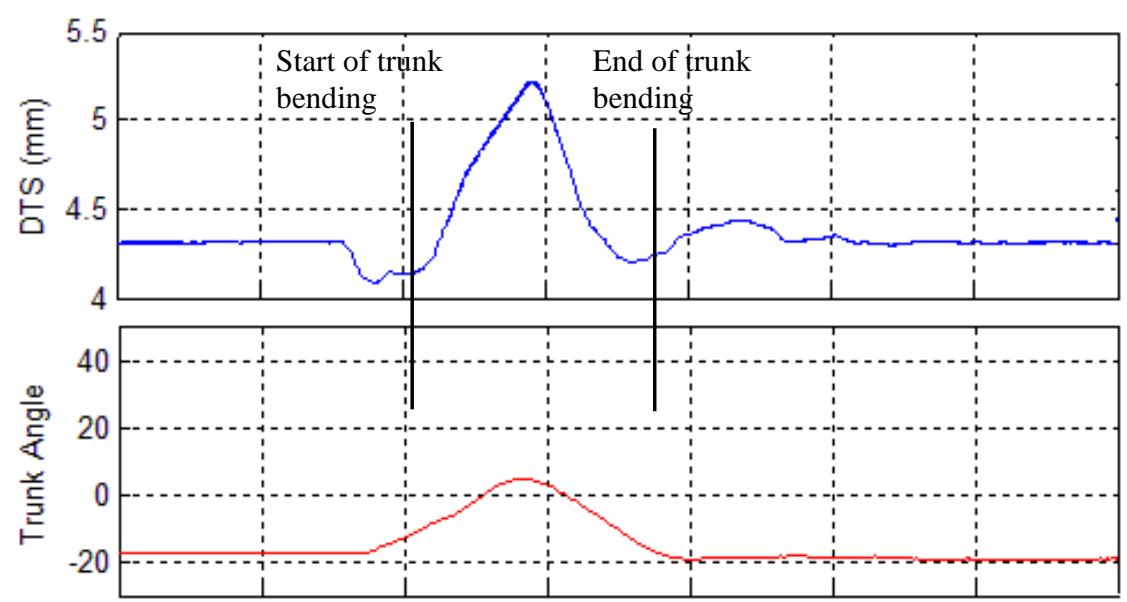

Figure 11 Time normalised DTS transient, motion captured body angles, of one stroke participant (S1) corresponding to a 'max reach' in the reaching task 


\begin{tabular}{|c|c|c|c|c|c|c|c|c|c|}
\hline \multirow{2}{*}{ Healthy Participants } & \multicolumn{9}{|c|}{ Averaged of 10 trials Trunk Angle (Degree) } \\
\hline & \multicolumn{3}{|c|}{$\begin{array}{c}\text { Condition A } \\
(120 \%)\end{array}$} & \multicolumn{3}{|c|}{$\begin{array}{c}\text { Condition B } \\
(150 \%)\end{array}$} & \multicolumn{3}{|c|}{$\begin{array}{l}\text { Condition C } \\
\text { (Max. reach) }\end{array}$} \\
\hline $\mathrm{H} 1$ & \multicolumn{3}{|c|}{0.4} & \multicolumn{3}{|c|}{28.4} & \multicolumn{3}{|c|}{61.4} \\
\hline $\mathrm{H} 2$ & \multicolumn{3}{|c|}{3} & \multicolumn{3}{|c|}{34.1} & \multicolumn{3}{|c|}{52.5} \\
\hline H3 & \multicolumn{3}{|c|}{1.5} & \multicolumn{3}{|c|}{22.3} & \multicolumn{3}{|c|}{48} \\
\hline $\mathrm{H} 4$ & \multicolumn{3}{|c|}{7.7} & \multicolumn{3}{|c|}{31} & \multicolumn{3}{|c|}{48.8} \\
\hline $\mathrm{H} 5$ & \multicolumn{3}{|c|}{2} & \multicolumn{3}{|c|}{20.6} & \multicolumn{3}{|c|}{60.4} \\
\hline H6 & \multicolumn{3}{|c|}{2} & \multicolumn{3}{|c|}{36.4} & \multicolumn{3}{|c|}{54.3} \\
\hline $\mathrm{H} 7$ & \multicolumn{3}{|c|}{8.4} & \multicolumn{3}{|c|}{34.2} & \multicolumn{3}{|c|}{60} \\
\hline $\mathrm{H} 8$ & \multicolumn{3}{|c|}{9.9} & \multicolumn{3}{|c|}{30.2} & \multicolumn{3}{|c|}{50.9} \\
\hline $\mathrm{H} 9$ & \multicolumn{3}{|c|}{2.1} & \multicolumn{3}{|c|}{26.8} & \multicolumn{3}{|c|}{51.8} \\
\hline $\mathrm{H} 10$ & \multicolumn{3}{|c|}{4.1} & \multicolumn{3}{|c|}{27.6} & \multicolumn{3}{|c|}{54.5} \\
\hline & Min & Avg & Max & Min & Avg & Max & Min & Avg & Max \\
\hline & 0.4 & 4.1 & 9.9 & 20.6 & 29.2 & 36.4 & 48 & 54.3 & 61.4 \\
\hline
\end{tabular}

Table 3 Trunk angle of healthy participants for the three reaching conditions obtained from the 3D motion capture system

The ANN described in section 2, developed from simulation data, was applied in the pilot study. To verify the results, a reference table is provided corresponding with trunk bending angle and the reaching conditions for healthy participants, summarized in Table 3. Condition A corresponds with an average trunk bending angle in the range from $0.4^{\circ}$ to $9.9^{\circ}$. Condition $\mathrm{B}$ corresponds with the average angle of $29.2^{\circ}$ (in the range of $20.6^{\circ}$ to $36.4^{\circ}$ ). Condition $\mathrm{C}$ corresponds with the average angle of 54.3 (in the range of $48^{\circ}$ to $61.4^{\circ}$ ).

\begin{tabular}{|c|c|c|c|c|}
\hline $\begin{array}{c}\text { Stroke } \\
\text { Participants }\end{array}$ & $\begin{array}{c}\text { Fugl-Meyer } \\
\text { score }\end{array}$ & $\begin{array}{c}\text { Average trunk angle } \\
\text { ( degree) to the } \\
\text { critical boundary }\end{array}$ & $\begin{array}{c}\text { Relevant } \\
\text { discrimination } \\
\text { condition }\end{array}$ & $\begin{array}{c}\text { Number of correct } \\
\text { classifications } \\
\text { (out of 15) }\end{array}$ \\
\hline S1 & $20 / 60$ & 21 & Condition B & 11 \\
\hline S2 & $58 / 60$ & 4.3 & Condition A & 15 \\
\hline S3 & $59 / 60$ & 4.3 & Condition A & 15 \\
\hline S4 & $59 / 60$ & 6 & Condition A & 15 \\
\hline S5 & $38 / 60$ & 30.9 & Condition B & 15 \\
\hline \multicolumn{5}{|c}{$\begin{array}{c}\text { AVERAGE ACCURACY } \\
\text { (71 correct classifications out of 75) }\end{array}$} \\
\hline \multicolumn{7}{|c}{$\mathbf{9 4 . 7 \%}$}
\end{tabular}

Table 4 Results of classifications of reaching to the critical boundary with the Artificial Neural Network

The accuracy of the ANN to discriminate the level of motor impairment in stroke participants is shown in Table 4. For participants S2, S3, and S4 of mild motor impairment (FM scores are above 50), the results show similarity with healthy participants (all classified as Condition A). For participants S1 and S5 of moderate impairment (FM scores are between 20 and 40), the critical boundary is classified as Condition B. The average accuracy of classifications in this trial is $94.7 \%$. It is identified that the accuracy for stroke participant S1 is $73.3 \%$ (11 out of 15 ), which is far below the accuracy for other participants at $100 \%$. The FM score of stroke participant $\mathrm{S} 1$ is 20 out of 60 , which is the lowest among all stroke participants. The second lowest score is 38 (participant S5). This suggests that to improve the performance of the ANN, further data will be needed for lower scored patients, and new patent inputs will need to be identified to deal with the complex range of movement illustrated in Figure 9.

\section{Discussion}

A tactile sensing scheme based on the distributive approach of mechanically coupled sensing elements has been implemented in a seat to investigate its application as a means to 
discriminate the classification of upper limb impairment for the stroke survivor. In a reaching task it was found that reliable discrimination could be achieved using a feedforward back propagation ANN. Mathematical modelling was used to train the ANN. The purpose was to test the robustness of ANN to alterations in anthropometric data and movement variability which would be present in assessing larger group of participants. Using 5 fundamental parameters describing the coupled sensory transients, training of the neural network was found to be efficient, robust and reliable with accuracy of automatic classification at $94.7 \%$ for survivors in the range of mild to moderate impairment. The performance of the approach to sensing is robust, with tolerance to both the variation in motion and seated position in the well-defined reaching task. The mechanically simple embodiment of device described in the form of a chair, uses four proximity sensors and demonstrates that simple approaches are feasible. The chair provides structured set-up and physical support for the survivor.

The results are encouraging. Despite its recognized limitations, the Fugl-Meyer test is widely used for the assessment of upper limb in stroke survivors and outcome of rehabilitation interventions (Singer \& Garcia-Vega; 2017). Our approach resulted in similar classification of upper limb functionality based on the Fugl-Meyer score. However, discrimination accuracy for S1 was low which affected overall accuracy of the discrimination process. Fugl-Meyer score for S1 was 20 out of 60 , which was the lowest amongst stroke participants. The second lowest score (S5 - 38) resulted in perfect discrimination. This suggested that to improve the performance of the ANN, not only further data would be needed for the low scored participants, but also new input patterns should be identified to train the networks which can also overcome some of the limitations of the Fugl-Meyer test (e.g. in identification of differences in the pattern of movements in those with similar score but different functionalities). Future experiments will examine simultaneous real-time discrimination of the trunk and arm motions. This is expected to be achievable and will provide the possibility of integration with a gaming environment suitable for the rehabilitation of stroke survivors (references here) and other individuals with upper limb motor impairments. Working from previous investigations, this is expected to be achievable and will provide for integration with a gaming environment and application to a wider range of stroke survivors with upper limb impairment.

\section{References}

1. Stroke statistics, http://www.stroke.org.uk/resource-sheet/stroke-statistics

2. Nasr, N., Torsi, S., Wright, P., Mawson, S., Mountain, G (2010) Supporting the selfmanagement of stroke by applying a user-centred design approach. Clinical Rehabilitation, 24: 276-287.

3. Murphy, Margit Alt, Carin Willén, and Katharina S. Sunnerhagen. "Kinematic variables quantifying upper-extremity performance after stroke during reaching and drinking from a glass." Neurorehabilitation and neural repair 25, no. 1 (2011): 71-80.

4. Volpe, B. T., H. I. Krebs, N. Hogan, L. Edelstein, C. Diels, and M. Aisen. "A novel approach to stroke rehabilitation Robot-aided sensorimotor stimulation."Neurology 54, no. 10 (2000): 1938-1944.

5. González-Ortega, D., F. J. Díaz-Pernas, Mario Martínez-Zarzuela, and Miriam AntónRodríguez. "A Kinect-based system for cognitive rehabilitation exercises monitoring." Computer methods and programs in biomedicine 113, no. 2 (2014): 620631.

6. Cho, Sangwoo, Jeonghun Ku, Yun Kyung Cho, In Young Kim, Youn Joo Kang, Dong Pyo Jang, and Sun I. Kim. "Development of virtual reality proprioceptive rehabilitation system for stroke patients." Computer methods and programs in biomedicine 113, no. 1 (2014): 258-265.

7. Andersson, Pontus, and Erika Franzén. "Effects of weight-shift training on walking ability, ambulation, and weight distribution in individuals with chronic stroke: a pilot study." Topics in stroke rehabilitation (2015): 1074935715Z-00000000052. 
8. Patterson, Kara K., Avril Mansfield, Louis Biasin, Karen Brunton, Elizabeth L. Inness, and William E. McIlroy. "Longitudinal changes in poststroke spatiotemporal gait asymmetry over inpatient rehabilitation." Neurorehabilitation and neural repair (2014): 1545968314533614.

9. Nikolay Mikov, "A distributive approach to tactile sensing for application to human movement", PhD thesis, Brunel University, 2015.

10. Yousef, Hanna, Mehdi Boukallel, and Kaspar Althoefer. "Tactile sensing for dexterous in-hand manipulation in robotics-A review." Sensors and Actuators A: physical 167, no. 2 (2011): 171-187.

11. Tiwana, Mohsin I., Stephen J. Redmond, and Nigel H. Lovell. "A review of tactile sensing technologies with applications in biomedical engineering." Sensors and Actuators A: physical 179 (2012): 17-31.

12. Xie, Hui, Allen Jiang, Lakmal Seneviratne, and Kaspar Althoefer. " Pixel-based optical fiber tactile force sensor for robot manipulation." In Sensors, 2012 IEEE, pp. 1-4.

13. Shigeyuki Shimachi1, Surakij Hirunyanitiwatna, Yasunori Fujiwara, Akira Hashimoto and Yoshinori Hakozaki, 'Adapter for contact force sensing of the da Vinci® robot', The International Journal of Medical Robotics and Computer Assisted Surgery, Volume 4, Issue 2, pages 121-130, June 2008

14. Hammond III, Frank L., Rebecca K. Kramer, Qian Wan, Robert D. Howe, and Robert J. Wood. "Soft Tactile Sensor Arrays for Force Feedback in Micromanipulation." IEEE SENSORS JOURNAL 14, no. 5 (2014): 1443.

15. R. Taylor, X. Du, D. Proops, A. Reid, C. Coulson, P.N. Brett,' A sensory guided surgical micro-drill', Proceedings of the Institution of Mechanical Engineers, Part C: Journal of Mechanical Engineering Science, Volume 224, Number 7, pages 1531-1537, 2010

16. B Tam, X Ma, P N Brett, An experimental investigation of a fibre optic sensing method to discriminate contact and motion of a flexible digit typical of invasive clinical environments, accepted, Part H: Journal of Engineering in Medicine 2009

17. Brett,P.N., Stone,R.S.W. and Evans,B.S., 'A system for the automatic handling of compact shaped dough products' ,Int J of Mechatronics, Vol 8, No.2, pp85-102, 1998

18. $\mathrm{Z} \mathrm{Wu}, \mathrm{X} \mathrm{Ma}, \mathrm{P} \mathrm{N}$ Brett and $\mathrm{J} \mathrm{Xu}$, Vibration analysis of submerged rectangular microplates with distributed mass loading, Proc. Royal Society, A, 8 April 2009 vol. 465 no. 2104, 1323-1336

19. M Elliott, X Ma, P N Brett, A smart sensing platform for the classification of ambulatory patterns, Proceedings of the Institution of Mechanical Engineers, Proc. IMechE, Part H: J. Engineering in Medicine, 2009, 223(H5), 567-575

20. Tongpadungrod,P., Rhys, D. and Brett,P.N.,' The optimisation of a distributive contact tactile sensing technique for measuring the position of a static point load.', International J of Sensors and Actuators, Part A, 105, 2003, pp47-54.

21. M. T. Elliott, I. Petra, X. Ma, P. N. Brett and D. J. Holding, Quantifying sway through surface deflection patterns: A novel approach using distributive tactile sensing, Part $\mathrm{H}$ : Journal of Engineering in Medicine, 2009, 223(H7), 903-912.

22. M. Jaderberg, K. Simonyan, A. Vedaldi, and A. Zisserman, "Synthetic data and artificial neural networks for natural scene text recognition," arXiv preprint arXiv:1406.2227, 2014.

23. M. Jaderberg, K. Simonyan, A. VedaldiA, and A. Zisserman, "Reading text in the wild with convolutional neural networks," International Journal of Computer Vision, vol. 116, no. 1, pp. 1-20, 2016.

24. A. Gupta, A. Vedaldi, and A. Zisserman, "Synthetic Data for Text Localisation in Natural Images," in Proceedings of the IEEE Conference on Computer Vision and Pattern Recognition, 2016, pp. 2315-2324.

25. Le TA, Baydin AG, Zinkov R, Wood F. Using synthetic data to train neural networks is model-based reasoning. InNeural Networks (IJCNN), 2017 International Joint Conference on 2017 May 14 (pp. 3514-3521).

26. Moate CP, Hayward SD, Ellis JS, Russell L, Timmerman RO, Lane RO, Strain TJ. Vehicle Detection in Infrared Imagery Using Neural Networks with Synthetic Training 
Data. InInternational Conference Image Analysis and Recognition 2018 Jun 27 (pp. 453461).

27. Plagenhoef S, Evans FG, Abdelnour T. Anatomical data for analyzing human motion. Research quarterly for exercise and sport. 1983 Jun 1; 54(2):169-78.

28. Materials Data Book. (2003). Cambridge University Engineering Department. 